\title{
Valve-sparing aortic root replacement in a patient with retroaortic course of the left circumflex artery
}

\author{
Rody Akiel ${ }^{1}$, Julien Guihaire ${ }^{1}$, Marc Antoine Isorni ${ }^{2}$, and Philippe Deleuze ${ }^{1}$ \\ ${ }^{1}$ Marie Lannelongue Hospital, Groupe Hospitalier Paris Saint Joseph \\ ${ }^{2}$ Marie Lannelongue Hospital, Groupe Hospitalier Paris Saint Joseph,
}

May 11, 2020

\begin{abstract}
Anomalous origin of the left circumflex artery (LCA) arising from the right coronary sinus was observed in a 45 year-old man with aortic root aneurysm. Valve-sparing aortic root replacement (VSARR) was decided despite the subannular course of the LCA. A modified Tirone David procedure was performed with specific consideration for distribution of the proximal suture line due to the peri-aortic and subannular course of the LCA. Due to the risk of LCA injury, a coronary artery bypass grafting was performed using the left internal thoracic artery to secure the perfusion of the LCA. The challenging association of aortic root aneurysm and anomalous origin and course of the LCA was managed successfully during VSARR.
\end{abstract}

\section{Introduction}

Valve-sparing aortic root replacement (VSARR) has become a standard of care for young patients with aortic root aneurysm. ${ }^{1}$ In this procedure, coronary arteries are detached from the aortic wall and reimplanted in their corresponding neosinuses. This approach may be challenging in presence of congenital coronary artery disease, as observed in about $1 \%$ of patients undergoing cardiac catheterization. ${ }^{2}$ We report specific considerations for VSARR related to the unusual association of aortic root aneurysm and anomalous origin of the left circumflex artery (LCA).

\section{Case description}

A 45 year-old male patient presented for a dilated aortic root depicted on a routine transthoracic echocardiography. The patient was asymptomatic and physical examination was unremarkable. Chest CT imaging confirmed the aortic aneurysm limited to the root with a maximal diameter of $51 \mathrm{~mm}\left(29.4 \mathrm{~mm} / \mathrm{m}^{2}\right)$. An abnormal origin of the LCA emerging from the right coronary sinus was remarkable. The aortic valve was tricuspid with mild central regurgitation. Preoperative coronary angiogram confirmed the aberrant origin of the LCA with a separate ostia from the right coronary artery (RCA) (Figure 1 ). The proximal segment of the LCA ran along the wall of the non-coronary sinus without evidence for intramural course and joined the left atrial roof at the level of the aortic annulus. The distal LCA thereafter reemerged in the left atrioventricular groove (Figure 1 ). The anatomy of the RCA and left anterior descending artery (LAD) was unremarkable. VSARR (David procedure) was decided for this young patient who expected to perform intensive physical activity. In the present case, the proximal subannular suture line during the David procedure may lead to irreversible injury of the retroaortic and subannular segment of LCA. ${ }^{3}$ A coronary artery bypass grafting (CABG) of the LCA combined with VSARR was therefore planned to secure the perfusion of the left ventricular lateral wall.

After midline sternotomy, the left internal thoracic artery was harvested as a skeletonized graft. Cardiopulmonary bypass $(\mathrm{CPB})$ was then established between the right atrium and the distal ascending aorta. 
Myocardial protection was obtained by antegrade mild hypothermic $(32 @ \mathrm{C})$ blood cardioplegia, repeated each 20 minutes. The aortic root was carefully dissected and the location of the coronary ostia was identified. The abnormal course of the LCA was isolated along the non-coronary sinus, confirming the absence of intramural path at this level (Figure 2 ). The dissection was conducted as low as possible to prepare for the proximal suture line of the aortic graft at the level of the ventriculo-aortic junction. The limit of the dissection was the roof of the left atrium, where the LCA deeply engaged towards the left atrioventricular groove. The CABG was then performed using the left internal thoracic artery to the first obtuse marginal artery. Afterwards, the aortic root was excised with preservation of two coronary buttons, one for the left main and a larger one for both the RCA and LCA ostia. The aortic valve was inspected and did not show any anatomical contraindication for VSARR procedure. A 30-mm gelatin-impregnated Valsalva prosthetic graft was first sutured using 9 interrupted U-stitches at the level of the ventriculo-aortic junction, avoiding to damage the retro-aortic course of the LCA. The native aortic valve was then included into the Valsalva tube using continuous running sutures. Inspection of the re-implanted aortic valve showed favorable leaflet coaptation (Figure 2 ). Reimplantation of coronary buttons began with the left main using continuous running suture. The coronary button including the RCA and LCA ostia was sutured to the right coronary sinus of the Valsalva graft avoiding any malposition or kinking of the proximal LCA. The peri-aortic course of the LCA was therefore respected along the new non-coronary sinus (Figure 2 ). The distal anastomosis between the Valsalva graft and the distal ascending aorta was completed during warming. Sinus rhythm at 75 bpm recovered spontaneously after aortic clamp release and CPB was weaned after 15 minutes of circulatory support. Aortic cross-clamping and CPB periods were 182 and 202 minutes respectively. Transesophageal echocardiographic examination showed satisfying aortic valve anatomy (effective height at $9 \mathrm{~mm}$; coaptation length at $5 \mathrm{~mm}$ ) without evidence for residual regurgitation. Both ventricles presented with normal motion, especially considering the left ventricular free wall.

Transient acute kidney injury related to hypovolemia was observed during the early postoperative course. There was no evidence for myocardial dysfunction, especially the echocardiography did not show abnormal motion of the left ventricular wall. The postoperative coronary angiogram showed normal LAD and RCA, as well as full patency of the LCA, especially at the level of her retro-aortic course (Figure 2 ). The patient recovered quickly from surgery and was able to resume professional activity 6 weeks after hospital discharge.

\section{Comment}

The main insights of the present case report are threefold. First, anatomical investigation of the coronary network is warranted before aortic root surgery, even in young patients without cardiovascular comorbidities. The association between aortic root aneurysm and coronary anomalies is scarcely reported in the literature. ${ }^{4}$ Anomalous origin and course of the LCA arising from the right coronary sinus is observed in 0.37 to $0.7 \%$ of patients undergoing cardiac catheterization. ${ }^{3,5}$ This anomaly has usually no clinical significance but should be investigated before aortic root surgery. The surgical procedure can therefore be planned to deal with this aberrant anatomical configuration. ${ }^{2}$ Second, this condition is challenging due to the peri-aortic and subannular course of the LCA since VSARR requires extensive dissection of the aortic root as low as possible. Moreover the David procedure needs sutures below the aortic annulus and may therefore be at risk for perioperative coronary injury in case of abnormal retro-aortic course of the LCA. The decision to perform David procedure for VSARR rather than aortic root remodeling was based on the better long-term outcomes associated with the reimplantation approach. The Yacoub procedure does not require a proximal suture line along the circumference of the ventriculo-aortic junction but has been associated with a higher rate of late reoperation ( $11 \%$ at 5 years) compared to the David procedure ( $<5 \%$ at 15 to 20 years). ${ }^{1,6}$ We therefore privileged the approach associated with the better long-term outcomes for our young patient. Last and consistent with Nezic et al., we suggested to perform a preventional CABG to the distal LCA to secure the perfusion of the left ventricular wall. ${ }^{7}$ This approach could also be applied in case of retro-aortic and subannular course of the LCA in patients referred for aortic valve replacement. ${ }^{8}$ Indeed all sutures located along the aortic annulus below the left coronary sinus could be at risk for LCA injury.

VSARR remains an effective option for aortic root aneurysm associated with anomalous origin and course of 
the LCA. In our case, the ectopic LCA was preserved without any iatrogenic damage after David procedure for aortic root replacement.

Author contributions: JG: concept/desing/data interpretation/drafting, RA: data interpretation/drafting the article, MAI: data interpretation, PD: revision.

\section{References}

1-David TE, David CM, Feindel CM, Manlhiot C, Reimplantation of the aortic valve at 20 years. $J$ Thorac Cardiovasc Surg. 2017;153:232-238.

2- Yamanaka O, Hobbs RE. Coronary artery anomalies in 126,595 patients undergoing coronary arteriography. Cathet Cardiovasc Diagn . 1990;21:28-40.

3- Angelini P, Velasco JA, Flamm S. Coronary anomalies: incidence, pathophysiology, and clinical relevance. Circulation . 2002;105:2449-54.

4- Hatemi AC, Tongut A, Özyedek Z, Çerezci İ, Özgöl İ, Perk Gürün H. Association between ascending aortic diameter and coronary artery dilation: a demographic data analysis. J Int Med Res . 2016;6:1349-1358.

5- Plastiras SC, Kampessi OS, Gotzamanidou M, Kastanis P. Anomalous origin of the left circumflex artery from the right coronary artery: a case report. Cases J . 2008;1:336.

6- Yacoub MH, Gehle P, Chandrasekaran V, Birks EJ, Child A, Radley-Smith R. Late results of a valvepreserving operation in patients with aneurysms of the ascending aorta and root. J Thorac Cardiovasc Surg . 1998;115(5):1080-1090.

7-Nezic DG. The left internal mammary artery to bypass an abnormal circumflex artery from right coronary sinus in a patient scheduled for root aneurysm. Interact Cardiovasc Thorac Surg . 2014;19:885.

8-Liebrich M, Tzanavaros I, Scheid M, Voth W, Doll KN, Hemmer WB. Aortic valve/root procedures in patients with an anomalous left circumflex coronary artery and a bicuspid aortic valve: anatomical and technical implications. Interactive Cardio Vascular and Thoracic Surgery.2015;21:114-116.

\section{Figure Legends}

Figure 1. (A, B) Preoperative coronary angiography showed anomalous origin and course of the LCA with a separate ectopic ostia rising from the right coronary sinus, independently from the RCA. (C, D) Cardiac CT scan illustrated the retro-aortic course of the ectopic LCA and its location close to the aortic annulus before reaching the left atrioventricular groove. Ao, aorta; PA, pulmonary artery.

Figure 2. (A) Peroperative view of the anomalous course of the LCA along the non-coronary sinus without intramural path. (B) Surgical view of the valve-sparing aortic root replacement after reimplantation of the aortic valve inside the prosthetic Valsalva graft. The peri-aortic course of the ectopic LCA was preserved. (C) No post-operative stenosis was remarkable in any segment of the LCA on the coronary angiogram. 

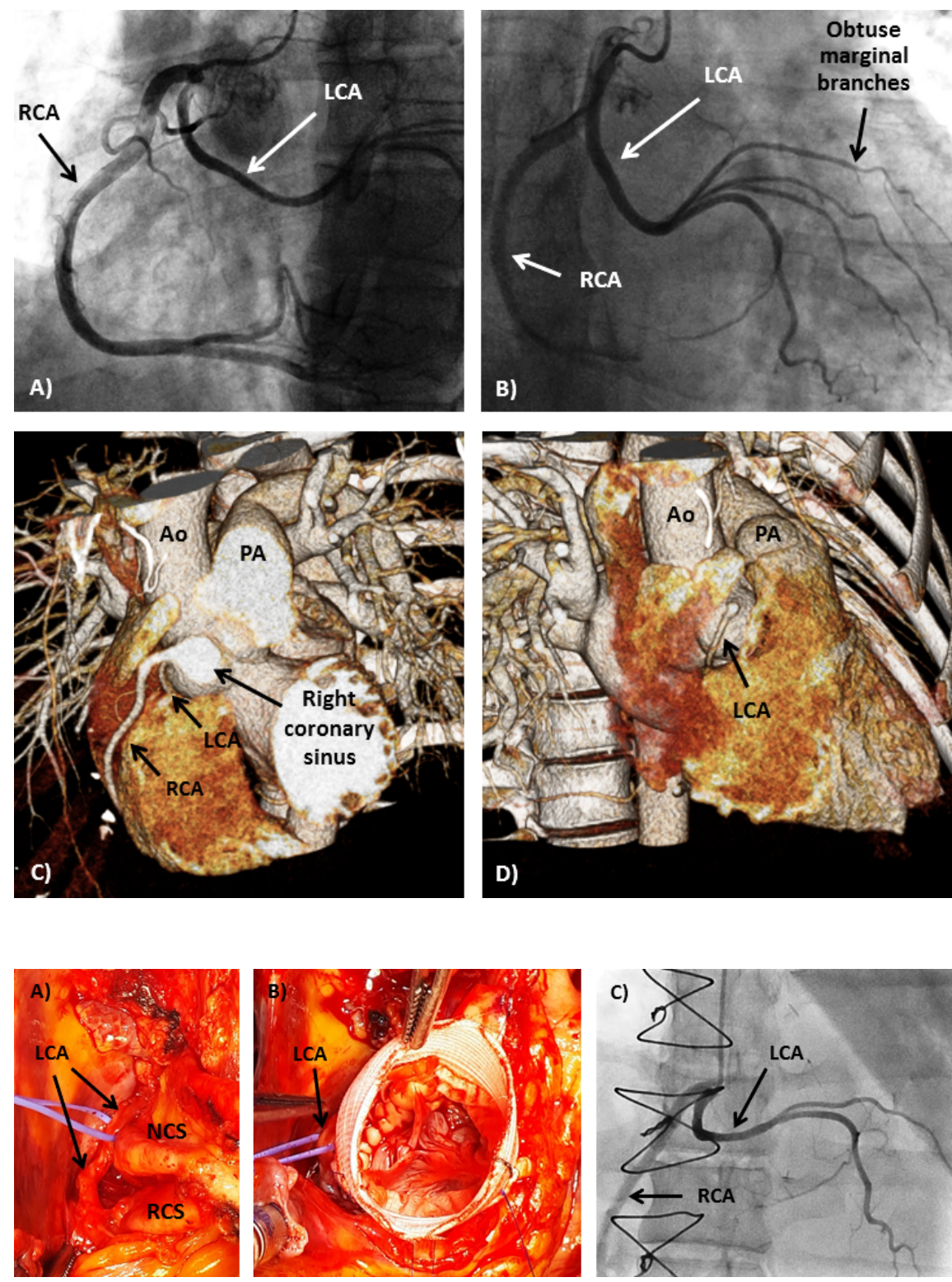